\title{
A method for tracking centre of mass displacement during non-seated cycling using an inertial sensor
}

\author{
Ross D. Wilkinson*, Glen A. Lichtwark \\ School of Human Movement and Nutrition Sciences, The University of Queensland, Australia
}

\begin{abstract}
Instantaneous crank power does not equal total joint power if a rider's centre of mass $(\mathrm{CoM})$ gains and loses mechanical energy. Thus, estimating CoM motion and the associated energy changes can provide valuable information about cycling performance. To date, an accurate and precise method for tracking CoM motion during outdoor cycling has not been validated. Purpose: To assess the suitability of an inertial measurement unit (IMU) for tracking CoM motion during non-seated cycling by comparing vertical displacement derived from an inertial sensor mounted to the lower back of the rider to an attached marker cluster and to a kinematic estimate of vertical CoM displacement from a full-body musculoskeletal model (Model). Methods: IMU and motion capture data were collected synchronously for 10 seconds while participants $(n=7)$ cycled on an ergometer in a non-seated posture at three power outputs and two cadences. A limits of agreement analysis, corrected for repeated measures, was performed on the range of vertical displacement between the IMU and the two other measures. A total of 303 crank cycles were analysed. Results: The IMU measured vertical displacement of the marker cluster with high accuracy $(1.6 \mathrm{~mm})$ and precision $(3.5 \mathrm{~mm})$ but substantially overestimated the kinematic estimate of rider CoM displacement. Conclusion: We interpret these findings as evidence that a single IMU placed on the lower back is unsuitable for tracking rider CoM displacement during non-seated cycling if the linearly increasing overestimation is unaccounted for.
\end{abstract}

Keywords: inertial measurement unit, motion analysis, joint power, bicycling

\section{Introduction}

During cycling, changes in mechanical energy of the rider's centre of mass (CoM) cause a discrepancy between instantaneous crank power and total joint power (van Ingen

Schenau \& Cavanagh, 1990). Research shows that a rider's CoM gains and loses mechan-

5 ical energy at rates of $4.5 \mathrm{~W} \cdot \mathrm{kg}^{-1}$ during non-seated cycling (Wilkinson et al. 2020a).

6 These changes in CoM mechanical energy increased peak crank force and peak crank

${ }^{*}$ Corresponding author. Phone: +17207274774

Email address: ross.wilkinson@uqconnect.edu.au (Ross D. Wilkinson) 
power. Thus, tracking a cyclist's CoM position provides valuable information about rider-bicycle interactions.

Optical motion capture systems provide a gold-standard measurement of a rider's CoM position during ergometer, treadmill, and over-ground cycling. But, there are limitations to each of these experimental setups. Ergometers allow the collection of many cycles per trial, but they constrain bicycle dynamics. This constraint alters the preferred movement pattern of the rider and may affect performance (Wilkinson et al. 2020b). Cycling on a treadmill provides ecological validity, but problems arise for some tasks such as sprinting. One problem is the danger of not matching belt velocity with wheel velocity if the rider accelerates. It is possible to capture over-ground cycling, but the calibrated volume of the camera system will limit the number of captured cycles. Thus, a method for tracking a rider's CoM motion when motion capture is not feasible would broaden the scenarios for examining rider-bicycle interactions.

Examining rider-bicycle interactions outside the laboratory is important because bicycle dynamics alter the preferred movement pattern of cyclists (Wilkinson et al., 2020b). For example, changes in CoM energy during non-seated cycling are greater when riders use a preferred amount of bicycle lean compared to when self-restricting lean (Wilkinson et al. 2020b). Research also shows that changes in CoM energy during non-seated ergometer cycling increase in response to increasing crank power and decreasing cadence (Wilkinson et al. 2020a). Thus, tracking a rider's CoM motion outside the laboratory would confirm whether these results extrapolate to over-ground cycling.

Full-body kinematic analyses provide accurate estimates of CoM displacement and velocity during non-seated cycling (Wilkinson et al., 2020a) and many other forms of locomotion (Gard et al., 2004: Hamner \& Delp, 2013). These analyses use segment positions and inertial parameters to compute CoM position and changes in total mechanical energy (potential + kinetic) (Eng \& Winter, 1993). Assuming constant mass, potential energy changes depend on vertical displacement, while kinetic energy changes depend on velocity in three dimensions. However, because the CoM location of standing humans is anterior to the lumbo-sacral joint, there is evidence that a single sacral marker provides a convenient and reliable approximation of vertical CoM displacement during walking (Gard et al., 2004, Thirunarayan et al., 1996, Saini et al., 1998). Pioneering research on non-seated cycling used a similar simplified method to estimate the pattern of vertical CoM displacement during non-seated cycling (Soden \& Adeyefa, 1979). Their results showed good agreement with more recent full-body kinematic analyses (Wilkinson et al., 2020b). Thus, tracking a single sacral marker may provide a suitable estimate of CoM position and mechanical energy during non-seated cycling.

Previous research has assessed the validity of using inertial measurement unit (IMU) data to provide an accurate measure of displacement and orientation of a point representing the CoM of a thoroughbred horse during locomotion (Pfau et al., 2005). When compared to optical motion capture, the sensor error for tracking the trunk movement of the horse in each axis was less than $5 \%$ during walking and $7 \%$ during a trot or canter. Here, we assess the validity of an IMU mounted near the sacrum for measuring vertical CoM displacement and associated energy changes of cyclists riding in a non-seated posture. Our assessment compares the derived vertical displacement of the IMU to an attached marker cluster; tracked with gold-standard optical motion capture technology, and to a full-body kinematic estimate of vertical CoM displacement. 


\section{Methods}

Seven recreational cyclists participated in this study ( 5 men and 2 women, age: $24 \pm 13$ yrs, height: $1.75 \pm 0.07 \mathrm{~m}$, mass: $71 \pm 11 \mathrm{~kg}$ ). Each participant gave written informed consent before participating in the study according to the procedures approved by the Human Ethics Committee of The University of Queensland.

\subsection{Experimental design}

Participants performed all trials on the same cycling ergometer (Excalibur Sport, Lode BV, Groningen, The Netherlands). The saddle height and handlebar position were matched to each participant's accustomed cycling position. Participants wore the same model of cycling shoes in their preferred shoe size (SH-R070, Shimano, Osaka, Japan) that clipped into the pedals (SH-R540, Shimano, Osaka, Japan).

At the beginning of the session, participants warmed-up by cycling for 5 minutes at $100 \mathrm{~W}$ at their preferred cadence. Participants then performed five maximal 5-s sprints in a seated posture, each separated by 3 minutes of rest. These sprints determined each participant's instantaneous maximal power output $\left(P_{\max . i}\right)$, which was used to individualise power output in the experimental trials. The experimental trials consisted of six 10 -s bouts of non-seated cycling at three different power outputs $(10 \%, 30 \%$, and $50 \%$ of $\left.P_{\operatorname{max.i}}\right)$ and two different cadences $(70 \mathrm{rpm}$ and $120 \mathrm{rpm}$ ), each separated by 3 minutes of rest. Each participant completed the experimental trials in a randomised order. For all experimental trials, the ergometer was set to Hyperbolic mode, which ensured that the power output remained constant independent of cadence. Thus, participants maintained the target cadence using feedback from the visual display on the ergometer for 10 seconds.

\subsection{Optical motion capture}

Reflective markers and lightweight marker clusters were secured to the skin using a combination of double-sided tape and self-adhesive bandage at previously described locations suitable for measuring full-body kinematics (Wilkinson et al., 2020c a) (marker locations are shown in Figure 1). The three-dimensional position of each marker was collected for 10 seconds at $200 \mathrm{~Hz}$ using an eight-camera, opto-electric motion capture system (Oqus, Qualisys AB, Gothenburg, Sweden). Motion capture data was processed using Qualisys Track Manager software (2019.1, Qualisys AB, Gothenburg, Sweden) before being exported to Matlab (R2020a, MathWorks Inc., Natick, MA).

\section{3. $I M U$}

An IMU (BlueThunder Sensor, iMeasureU, Auckland, New Zealand) attached to a rigid cluster of reflective markers was secured to the rider's skin at the intersection of Tuffier's line and the midline of the lumbar spine ( L4 spinous process) using doublesided tape (See Figure1). The markers were attached such that the plane created by the markers corresponded to the $X Y$ plane of the IMU. A self-adhesive bandage was wrapped around the cluster and torso of the rider to limit soft-tissue artefact. The IMU Research Application (IMeasureU, Auckland, New Zealand) was used to collect IMU data at 100 $\mathrm{Hz}$ via Bluetooth to an iPad (Apple, California, United States). The IMU contained a triaxial accelerometer $( \pm 16 \mathrm{~g})$, triaxial gyroscope $\left( \pm 2000^{\circ} \mathrm{s}^{-1}\right)$, and triaxial magnetometer $( \pm 1200 \mu T)$ with micro-electro-mechanical systems (MEMS) technology connected to a 
small circuit board. Thus, the sensor logged acceleration, angular velocity, and magnetic flux data in the three orthogonal planes. IMU and motion capture data were synced by tapping the sensor before the start of the trial with a motion capture calibration wand. The collision between the sensor and wand marker provided a synchronised spike in their respective resultant accelerations.

The approach used in this study for calculating orientation and linear displacement of the IMU have been described previously (Pfau et al. 2005$)$. Based on previous research we expected the inertial sensor to accurately track the attached marker cluster (Madgwick et al., 2011, Pfau et al. 2005), hence only one model of IMU was tested. However, the IMU used here did not output orientation, and hence we used a freely available sensor fusion algorithm to determine sensor orientation (Madgwick et al., 2011). We rotated the IMU data about the vertical axis so that the heading direction matched the motion capture system in the static trial, thereby aligning the orientation of the two systems. During the cycling trials, the quaternion orientation was used to rotate the linear acceleration and angular velocity of the IMU from its local-coordinate system to the global-coordinate system defined by gravity and due north. Acceleration due to gravity was removed from the vertical component of the acceleration signal. The linear accelerations were double integrated to calculate velocity and displacement. A short window (one cycle) was implemented for drift correction under the assumption that there would only be small cycle-to-cycle variations in movement pattern because participants were cycling at a constant power output and cadence. At each time point, the position of the rigid cluster was approximated by creating a virtual marker in the centre of the cluster; calculated as the mean position of the cluster markers. The notation used in this study has been provided in Table 1 .

[Figure 1 about here.]

\subsection{Musculoskeletal model}

Kinematic analysis was performed using a previously developed full-body musculoskeletal model (Rajagopal et al. 2016) within OpenSim software (Delp et al., 2007). A static trial was collected with each participant standing in a standard-anatomical posture to scale the model's segment lengths and inertial parameters to each participant. The scaled model and motion capture data were used to run inverse kinematics within OpenSim, which determined the whole-body CoM location at each frame. A detailed description of the scaling and inverse kinematic process can be found elsewhere (Wilkinson et al., 2020c).

\subsection{Statistical analysis}

Sensor performance was quantified for each experimental condition as the root-meansquare (RMS) error in each Euler parameter describing the yaw, pitch, and roll components of the IMU angular velocity compared to the attached marker cluster body. For each trial, the mean RMS error was calculated from the RMS error in each frame over the 10 seconds of data.

Agreement in the range of vertical displacement between the IMU and the two other measures was assessed within each condition by applying a Limits of Agreement (LoA) approach (Bland \& Altman, 1999). LoA analyses were corrected for repeated measures and encompassed accuracy (Bias), precision (standard deviation), average error $\left(\frac{\text { bias }}{\text { range }}\right)$, 
and maximum error ( $\left.\frac{\text { standard deviation }}{\text { range }}\right)$. Different LoA calculations were carried out depending on whether a significant linear trend was identified in the distribution of differences between the IMU and the two other measures (IMU - measure) as a function of the mean range of vertical displacement $\left(\frac{I M U+\text { measure }}{2}\right)$. If a linear trend was identified, bias was calculated as the equation of the linear model, rather than a constant value (Bland \& Altman, 2007). Sphericity of the data was checked using Mauchly's test and non-parametric analyses were used when necessary.

A simple linear regression was performed across all experimental conditions to identify the linear trend between the range of vertical IMU displacement to the two other measures. All statistical analyses were performed in Matlab.

[Table 1 about here.]

[Figure 2 about here.]

\section{Results}

To avoid any bias between the two cadence conditions at each power output, the number of cycles analysed from each participant was matched between all conditions. This meant that eight crank cycles were processed for each of the seven participants in each of the six conditions. Due to data dropout, 12 cycles were discarded, while a further 21 cycles were identified as outliers ( $>3$ scaled median absolute deviations from the median) (Leys et al. 2013) and removed from the analysis. Thus, a total of 303 cycles were analysed ( $\sim 7$ cycles per condition per participant). The distribution of differences between the IMU and the two other measures violated Mauchly's test for sphericity, thus non-parametric analyses were performed.

\subsection{Sensor performance}

The results of the sensor performance analysis are summarised in Table 2 Group mean $( \pm$ SD) RMS errors between the IMU and marker cluster body are reported for each condition. The data shown in Figure 2 illustrates how well the IMU was able to track the orientation and motion of the marker cluster.

\subsection{IMU vs optical motion capture}

Linear regression results and Bland-Altman plots are presented in Figure 4A and $\mathrm{B}$, respectively. Across all conditions, there was high agreement between the IMU and marker cluster range of vertical displacement (See Table 3). Differences between the IMU and marker cluster's range of vertical displacement showed no significant linear trend. On average, the IMU overestimated the cluster results by $1.6 \pm 3.5 \mathrm{~mm}$ (accuracy \pm precision), which equated to an average error of $1.8 \pm 3.9 \%$. Figure 3 illustrates how well the IMU was able to track the marker cluster's vertical displacement. 


\subsection{IMU vs musculoskeletal model}

Linear regression results and Bland-Altman plots are presented in Figure $4 \mathrm{C}$ and D, respectively. Across all conditions, agreement between the IMU and marker cluster range of vertical displacement decreased at higher ranges of vertical displacement (See Table 3 . Differences between the IMU and musculoskeletal model's range of vertical displacement showed a significant linear trend. Across all conditions, the IMU overestimated the model's results, especially at higher ranges of vertical displacement. Figure 3 illustrates the discrepancy between the IMU's vertical displacement and the model's vertical CoM displacement.

[Figure 3 about here.]

[Table 2 about here.]

[Figure 4 about here.]

[Table 3 about here.]

\section{Discussion}

We found that the IMU overestimated the kinematic estimate of vertical CoM displacement and that this overestimation increased linearly as the measured range of vertical CoM displacement increased. The IMU identified the trend that the range of a rider's vertical CoM displacement during non-seated cycling increases in response to increasing power output and at lower cadence, however, it underestimated the rate of this increase compared to full-body kinematics. Thus, the IMU is likely to be more accurate at lower power outputs when less CoM displacement occurs. However, the non-seated posture is typically used for producing high-power outputs during climbing and sprinting.

We interpret our findings as evidence that a single IMU can track the vertical displacement of an attached marker cluster with high accuracy and precision, but unacceptable discrepancies exist between the vertical displacement of the IMU and the kinematic estimate of rider CoM displacement. Discrepancies between the single IMU and rider CoM increased with increasing power output and decreasing cadence, but these increases were systematic and may be able to be accounted for using linear regression.

The overestimation of the kinematic estimate of rider CoM displacement suggests that a rider's lower back goes through a larger range of vertical displacement during each crank cycle than their CoM. This makes intuitive sense as the motion of body segments pther than the torso have a significant influence on the whole-body CoM (Kautz \& Neptune, 2002). Based on our findings, it appears that the difference between lower back and CoM displacement increases at higher power outputs. This suggests that negative work done by the arms (Turpin et al., 2016, Wilkinson et al., 2020a) likely acts on the torso to decouple lower back movement from the torso CoM. Thus, a single IMU on the lower back cannot account for the entirety of changes in CoM position due to motion of the legs and rotation of the torso.

We cannot rule out that a portion of the disagreement between the IMU and model was due to the processing of the IMU data, however, the accuracy between the IMU and cluster suggests that processing errors were minimal. It is possible that small offsets 
were present due to the manual synchronisation process between the IMU and motion capture data, which could be avoided in future studies by using hardware-synchronised systems. Another possible source of error was the misalignment between the IMU and marker cluster. The IMU was attached using a silicone putty which prevented any relative movement but does not guarantee that the alignment to the plane created by the marker clusters was exact. A misalignment between the IMU and marker cluster would cause a constant offset in the orientation data, however, small errors in orientation are known to have a minimal influence on linear displacements in a global-coordinate system (Pfau et al., 2005).

It is possible that multiple IMUs may provide a better estimate of a rider's CoM displacement, however, any additional weight or restriction of movement cause by such a system would likely prevent its widespread use. It is also possible that another location on the body may provide superior tracking of the CoM compared to the lower back. In general, the location of the CoM during non-seated cycling is in front of the pelvis and outside the body (See Figure 11). Thus, an IMU placed on the front of the pelvis would be closer to the CoM, however, this location would still not account for the influence of leg motion on CoM movement. Furthermore, we suspect that this location may be more likely to suffer from greater soft-tissue movement not related to CoM movement (Riddick \& Kuo, 2016). A single IMU placed on the lower back may still be suitable for other applications in the analysis of cycling. For example, a single IMU is lightweight and lowcost, meaning it could provide an accessible solution for identifying when a cyclist changes their posture from seated to non-seated. Our data suggest that the precision of the IMU would be adequate for providing within-condition comparisons. Given the accuracy and precision of IMUs for measuring the orientation and displacement of attached objects, a single IMU attached to the frame of a bicycle would be suitable for measuring its angular displacement and velocity.

\section{Conclusion}

We found that during non-seated cycling, a single IMU placed on the lower back overestimates a rider's vertical CoM displacement. As the range of CoM displacement increases, the overestimation error increases linearly. We interpret these findings as evidence that a single IMU placed on the lower back is unsuitable for tracking rider CoM displacement during non-seated cycling if the linearly increasing overestimation is not accounted for. Further development of a single-IMU method for measuring a rider's CoM displacement and associated mechanical energy fluctuations during over-ground cycling remains a future goal.

\section{Conflict of interest statement}

No conflict of interest to disclose.

\section{Acknowledgements}

R.D.W. was supported by an Australian Government Research Training Program Scholarship. 


\section{References}

Bland, J. M., \& Altman, D. G. (1999). Measuring agreement in method comparison studies. Statistical Methods in Medical Research, 8, 135-160. doi 10.1002/sim.5955

Bland, J. M., \& Altman, D. G. (2007). Agreement between methods of measurement with multiple observations per individual. Journal of Biopharmaceutical Statistics, 17, 571-582. doi 10.1080/ 10543400701329422

Delp, S. L., Anderson, F. C., Arnold, A. S., Loan, P., Habib, A., John, C. T., Guendelman, E., \& Thelen, D. G. (2007). OpenSim: Open source to create and analyze dynamic simulations of movement. IEEE transactions on bio-medical engineering, 54, 1940-1950. doi 10.1109/TBME.2007.901024.

Eng, J. J., \& Winter, D. A. (1993). Estimations of the horizontal displacement of the total body centre of mass: considerations during standing activities. Gait and Posture, 1, 141-144.

Gard, S. A., Miff, S. C., \& Kuo, A. D. (2004). Comparison of kinematic and kinetic methods for computing the vertical motion of the body center of mass during walking. Human Movement Science, 22, 597-610. doi 10.1016/j.humov.2003.11.002

Hamner, S. R., \& Delp, S. L. (2013). Muscle contributions to fore-aft and vertical body mass center accelerations over a range of running speeds. Journal of Biomechanics, 46, 780-787. doi 10.1016/j. jbiomech.2012.11.024 arXiv:NIHMS150003

Kautz, S. A., \& Neptune, R. R. (2002). Biomechanical determinants of pedaling energetics: internal and external work are not independent. Exercise and sport sciences reviews, 30, 159-65. doi 10.1097/ 00003677-200210000-00004.

Leys, C., Klein, O., Bernard, P., \& Licata, L. (2013). Detecting outliers: Do not use standard deviation around the mean, use absolute deviation around the median. Journal of Experimental Social Psychology, 49, 764-766.

Madgwick, S. O., Harrison, A. J., \& Vaidyanathan, R. (2011). Estimation of IMU and MARG orientation using a gradient descent algorithm. IEEE International Conference on Rehabilitation Robotics, (pp. 1-7). doi 10.1109/ICORR.2011.5975346

Pfau, T., Witte, T. H., \& Wilson, A. M. (2005). A method for deriving displacement data during cyclical movement using an inertial sensor. The Journal of experimental biology, 208, 2503-14. doi $10.1242 /$ jeb.01658.

Rajagopal, A., Dembia, C., DeMers, M., Delp, D., Hicks, J., \& Delp, S. (2016). Full body musculoskeletal model for muscle-driven simulation of human gait. IEEE Transactions on Biomedical Engineering, 63, 2068-2079. doi 10.1109/TBME. 2016.2586891.

Riddick, R. C., \& Kuo, A. D. (2016). Soft tissues store and return mechanical energy in human running. Journal of Biomechanics, 49, 436-441. doi 10.1016/j.jbiomech.2016.01.001

Saini, M., Kerrigan, D. C., Thirunarayan, M. A., \& Duff-Raffaele, M. (1998). The vertical displacement of the center of mass during walking: A comparison of four measurement methods. Journal of Biomechanical Engineering, 120, 133-139. doi 10.1115/1.2834293

Soden, P., \& Adeyefa, B. (1979). Forces applied to a bicycle during normal cycling. Journal of Biomechanics, 12, 527-541. doi 10.1016/0021-9290(79)90041-1

Thirunarayan, M. A., Kerrigan, D. C., Rabuffetti, M., Della Croce, U., \& Saini, M. (1996). Comparison of three methods for estimating vertical displacement of center of mass during level walking in patients. Gait and Posture, 4, 306-314. doi 10.1016/0966-6362(95)01058-0

Turpin, N. A., Costes, A., Moretto, P., \& Watier, B. (2016). Upper limb and trunk muscle activity patterns during seated and standing cycling. Journal of Sports Sciences, 35, 557-564.

van Ingen Schenau, G. J., \& Cavanagh, P. R. (1990). Power equations in endurance sports. Journal of Biomechanics, 23, 865-881.

Wilkinson, R. D., Cresswell, A. G., \& Lichtwark, G. A. (2020a). Riders Use Their Body Mass to Amplify Crank Power during Nonseated Ergometer Cycling. Medicine and Science in Sports and Exercise, Published Ahead of Print. doi 10.1249/MSS.0000000000002408.

Wilkinson, R. D., Cresswell, A. G., \& Lichtwark, G. A. (2020b). Rock and roll: The influence of bicycle lean on the mechanics of non-seated cycling. doi $10.31219 /$ osf .io/bfdtv

Wilkinson, R. D., Lichtwark, G. A., \& Cresswell, A. G. (2020c). The Mechanics of Seated and Nonseated Cycling at Very-High-Power Output: A Joint-level Analysis. Medicine and Science in Sports and Exercise, 52, 1585-1594. doi 10.1249/MSS.0000000000002285 


\section{List of Figures}

$1 \quad$ A single inertial sensor was used to estimate rider CoM displacement during non-seated cycling. A. An IMU attached to a rigid marker cluster was secured to the skin at the intersection of Tuffier's line (dashed) and lumbar spine midline ( L4 spinous process). B. Sagittal plane view of a scaled musculoskeletal model cycling in a non-seated posture. The kinematic estimate of the rider's CoM position is represented by the green sphere. . 10

2 The orientation of the sensor was matched closely with the attached marker cluster. Comparison of Euler angle and angular velocity parameters between the IMU and rigid marker cluster body for a single participant cycling in a non-seated posture at $30 \% \mathrm{P}_{\max . i}$ at $70 \mathrm{rpm}$. All angle values are in radians and angular velocity values are in radians per second. . . . 11

3 The pattern of vertical IMU displacement matched well with the marker cluster and model's CoM. Group mean vertical displacement of the IMU, marker cluster body, and CoM of the musculoskeletal model with respect to crank angle $\left(0^{\circ}\right.$ and $360^{\circ}=$ top dead centre) during non-seated cycling at three power outputs $\left(10 \%\right.$ (A,D), 30\% (B,E), and $50 \%$ (C,F) $\left.P_{\operatorname{max.}}\right)$ at $70 \mathrm{rpm}(\mathrm{A}-\mathrm{C})$ and $120 \mathrm{rpm}(\mathrm{D}-\mathrm{F}) . \ldots \ldots \ldots \ldots . \ldots . \ldots . \ldots 12$

4 The IMU tracked the cluster with high accuracy, but overestimated the model's range of vertical CoM displacement. A. Regression (left) and Bland-Altman plots (right) of the range IMU vertical displacement within each crank cycle as a function of Cluster vertical displacement (top) and Model CoM vertical displacement (bottom). The non-parametric repeatability coefficient $\left(R P C_{n p}\right)$ is also shown as a percentage of mean displacement. 


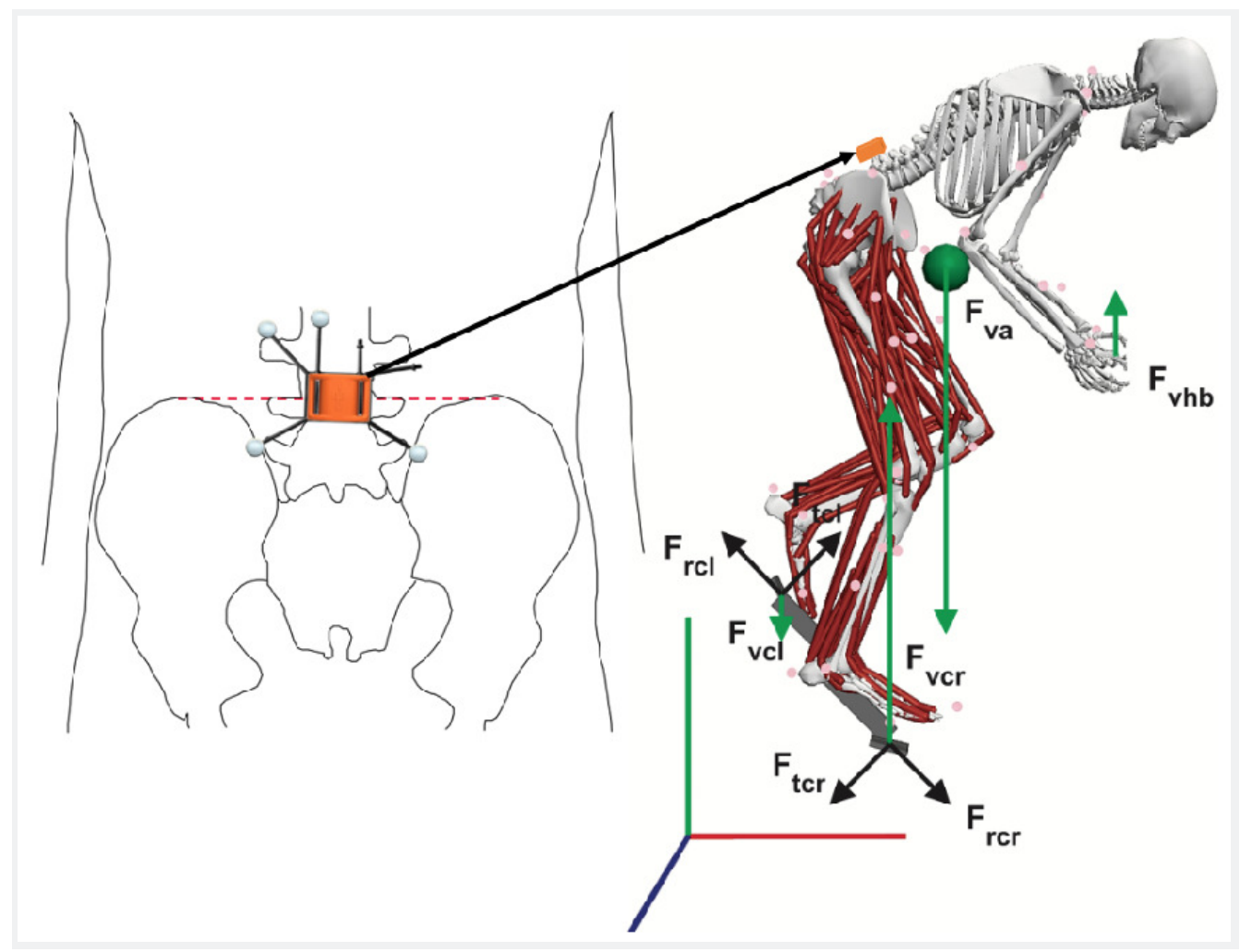

Figure 1: A single inertial sensor was used to estimate rider CoM displacement during non-seated cycling. A. An IMU attached to a rigid marker cluster was secured to the skin at the intersection of Tuffier's line (dashed) and lumbar spine midline ( $\sim \mathrm{L} 4$ spinous process). B. Sagittal plane view of a scaled musculoskeletal model cycling in a non-seated posture. The kinematic estimate of the rider's CoM position is represented by the green sphere. 


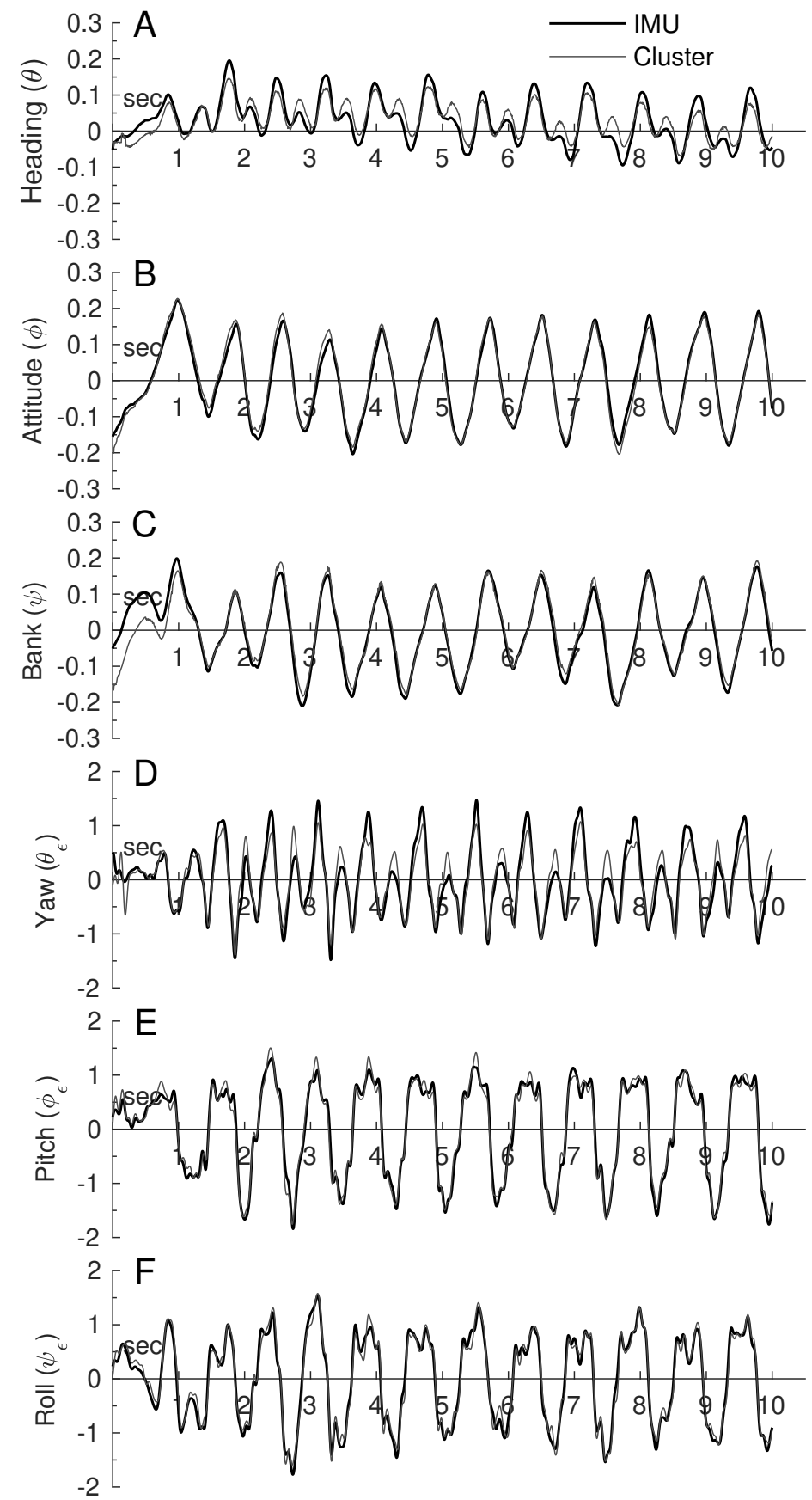

Figure 2: The orientation of the sensor was matched closely with the attached marker cluster. Comparison of Euler angle and angular velocity parameters between the IMU and rigid marker cluster body for a single participant cycling in a non-seated posture at $30 \% \mathrm{P}_{\operatorname{max.i}}$ at $70 \mathrm{rpm}$. All angle values are in radians and angular velocity values are in radians per second. 


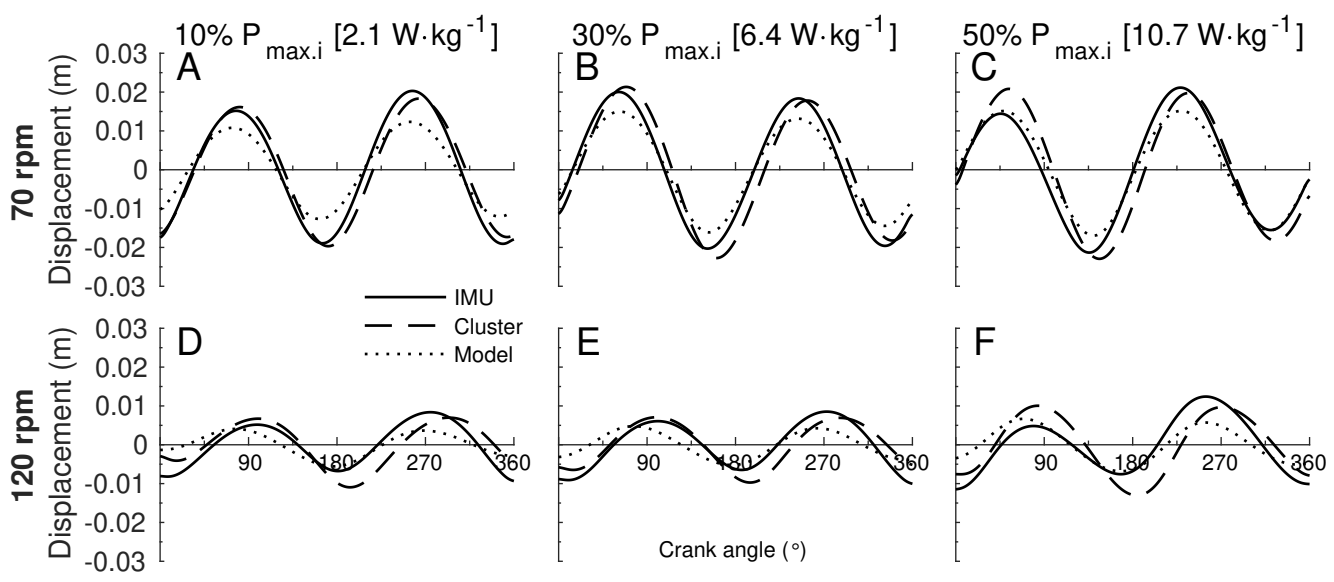

Figure 3: The pattern of vertical IMU displacement matched well with the marker cluster and model's CoM. Group mean vertical displacement of the IMU, marker cluster body, and CoM of the musculoskeletal model with respect to crank angle $\left(0^{\circ}\right.$ and $360^{\circ}=$ top dead centre $)$ during non-seated cycling at three power outputs $\left(10 \%(\mathrm{~A}, \mathrm{D}), 30 \%(\mathrm{~B}, \mathrm{E})\right.$, and $\left.50 \%(\mathrm{C}, \mathrm{F}) P_{\max . i}\right)$ at $70 \mathrm{rpm}(\mathrm{A}-\mathrm{C})$ and $120 \mathrm{rpm}$ (D-F). 

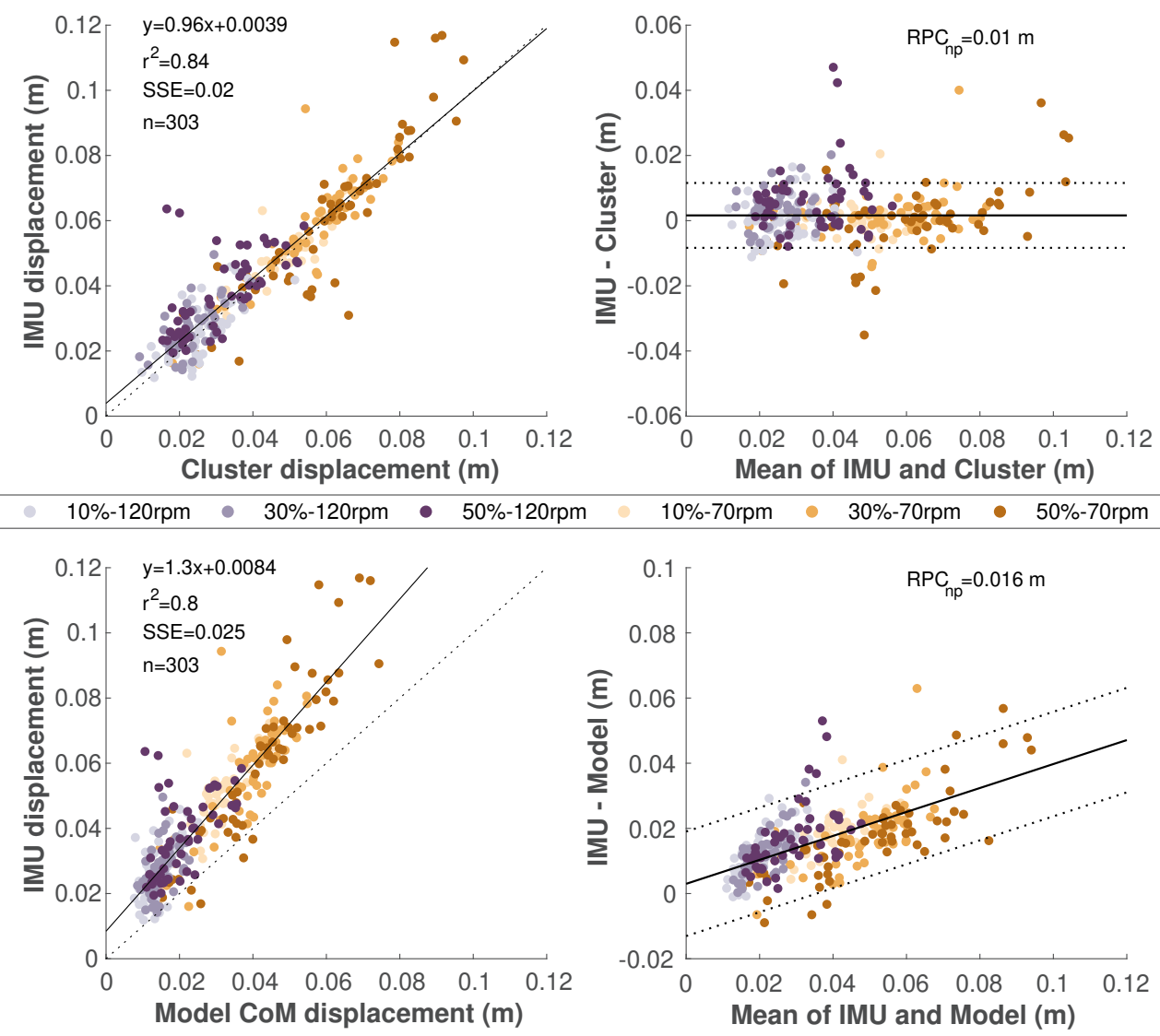

Figure 4: The IMU tracked the cluster with high accuracy, but overestimated the model's range of vertical CoM displacement. A. Regression (left) and Bland-Altman plots (right) of the range IMU vertical displacement within each crank cycle as a function of Cluster vertical displacement (top) and Model CoM vertical displacement (bottom). The non-parametric repeatability coefficient $\left(R P C_{n p}\right)$ is also shown as a percentage of mean displacement. 


\section{List of Tables}

1 Notation, reference coordinates, and definitions used to describe the orientation and motion of the IMU and marker cluster body in this study. . 15

2 The sensor performance was excellent across all conditions. Group mean ( \pm standard deviation) RMS error in each Euler parameter describing the yaw, pitch, and roll components of the IMU angular velocity ( rad . $s^{-1}$ ) compared to the rigid marker cluster body during non-seated cycling at three different power outputs $\left(10 \%, 30 \%\right.$, and $\left.50 \% P_{\operatorname{max.i}}\right)$ and two different cadences $(70 \mathrm{rpm}$ and $120 \mathrm{rpm}) . \ldots \ldots \ldots . \ldots 16$

3 There was good agreement between the IMU and cluster, but not between the IMU and model. A fixed limits of agreement analysis was used to compare the range of vertical displacement derived from an IMU to a rigid marker cluster. Positive values indicate an overestimation of the marker cluster's vertical displacement by the IMU (IMU - Cluster). All measures are in millimetres. A variable limits of agreement analysis was used to compare the range of vertical displacement derived from an IMU to the musculoskeletal model. All measures are in millimetres. . . . . . . . 17 
Parameter

Local- and global-coordinate system

Global $\mathrm{x}, \mathrm{y}$, and $\mathrm{z}$ axis definitions

Euler angles

Symbols

Angular velocity

Euler angle rotation order

Angle units

\section{Definitions and symbols}

right-handed cartesian

$+x$ to the right, $+y$ straight up,$+z$ toward viewer angular position about local $x, y$, and $z$ axes defined as bank, heading, and attitude, respectively $\psi=$ bank, $\theta=$ heading, $\phi=$ attitude about local $x, y$, and $z$ axes defined as roll $\left(\psi_{\epsilon}\right)$, yaw $\left(\theta_{\epsilon}\right)$, and pitch $\left(\phi_{\epsilon}\right)$, respectively

Heading, attitude, then bank radians and radians per second

Table 1: Notation, reference coordinates, and definitions used to describe the orientation and motion of the IMU and marker cluster body in this study. 


\begin{tabular}{|c|c|c|c|}
\hline \multirow[b]{2}{*}{ Conditions } & \multicolumn{3}{|c|}{ RMS error } \\
\hline & Yaw $\left(\theta_{\epsilon}\right)$ & $\operatorname{Pitch}\left(\phi_{\epsilon}\right)$ & $\operatorname{Roll}\left(\psi_{\epsilon}\right)$ \\
\hline \multicolumn{4}{|l|}{$10 \% P_{\max . i}$} \\
\hline $70 \mathrm{rpm}$ & $0.16 \pm 0.02$ & $0.14 \pm 0.02$ & $0.11 \pm 0.03$ \\
\hline $120 \mathrm{rpm}$ & $0.22 \pm 0.03$ & $0.17 \pm 0.03$ & $0.18 \pm 0.08$ \\
\hline \multicolumn{4}{|l|}{$30 \% P_{\max . i}$} \\
\hline $70 \mathrm{rpm}$ & $0.19 \pm 0.05$ & $0.15 \pm 0.04$ & $0.15 \pm 0.08$ \\
\hline $120 \mathrm{rpm}$ & $0.19 \pm 0.05$ & $0.18 \pm 0.08$ & $0.16 \pm 0.02$ \\
\hline \multicolumn{4}{|l|}{$50 \% P_{\max . i}$} \\
\hline $\begin{array}{c}70 \mathrm{rpm} \\
\ldots .\end{array}$ & $\begin{array}{c}0.21 \pm 0.02 \\
\ldots \ldots \ldots\end{array}$ & $\begin{array}{c}0.15 \pm 0.02 \\
\ldots\end{array}$ & $\begin{array}{c}0.12 \pm 0.01 \\
\cdots \cdots \cdots\end{array}$ \\
\hline $120 \mathrm{rpm}$ & $0.24 \pm 0.06$ & $0.19 \pm 0.05$ & $0.17 \pm 0.05$ \\
\hline
\end{tabular}

Table 2: The sensor performance was excellent across all conditions. Group mean ( \pm standard deviation) RMS error in each Euler parameter describing the yaw, pitch, and roll components of the IMU angular velocity $\left(\mathrm{rad} \cdot \mathrm{s}^{-1}\right)$ compared to the rigid marker cluster body during non-seated cycling at three different power outputs $\left(10 \%, 30 \%\right.$, and $\left.50 \% P_{\max . i}\right)$ and two different cadences $(70 \mathrm{rpm}$ and $120 \mathrm{rpm})$. 


\begin{tabular}{|c|c|c|c|c|c|c|c|}
\hline & \multicolumn{4}{|c|}{ Agreement analysis } & \multicolumn{3}{|c|}{ Descriptive statistics } \\
\hline & LloA & UloA & $\begin{array}{l}\text { Accuracy } \\
\text { (Bias) }\end{array}$ & $\begin{array}{l}\text { Precision } \\
\text { (MAD) }\end{array}$ & Range & $\begin{array}{l}\text { Ave. } \\
\text { err. }\end{array}$ & $\begin{array}{l}\text { Max. } \\
\text { err. }\end{array}$ \\
\hline $\begin{array}{l}\text { IMU vs } \\
\text { Cluster }\end{array}$ & -8.3 & 11.6 & 1.6 & 3.5 & 88.3 & $1.8 \%$ & $\pm 3.9 \%$ \\
\hline $\begin{array}{l}\text { IMU vs } \\
\text { Model }\end{array}$ & $\begin{array}{l}0.37 x- \\
13.1\end{array}$ & $\begin{array}{l}0.37 x- \\
19.0\end{array}$ & $\begin{array}{l}0.37 x+ \\
3.0\end{array}$ & 5.6 & - & - & - \\
\hline
\end{tabular}

Table 3: There was good agreement between the IMU and cluster, but not between the IMU and model. A fixed limits of agreement analysis was used to compare the range of vertical displacement derived from an IMU to a rigid marker cluster. Positive values indicate an overestimation of the marker cluster's vertical displacement by the IMU (IMU - Cluster). All measures are in millimetres. A variable limits of agreement analysis was used to compare the range of vertical displacement derived from an IMU to the musculoskeletal model. All measures are in millimetres. 